L'intégration régionale de la France dans le Pacifique océanien, une diplomatie multimodale

Regional Integration of France in the Oceanian Pacific: Multimodal Diplomacy

\title{
Christian Lechervy
}

\section{OpenEdition}

Journals

Édition électronique

URL : http://journals.openedition.org/jso/7282

DOI : $10.4000 /$ jso.7282

ISSN : $1760-7256$

Éditeur

Société des océanistes

Édition imprimée

Date de publication : 15 juin 2015

Pagination : 105-121

ISBN : 9782854301250

ISSN : 0300-953x

Référence électronique

Christian Lechervy, «L'intégration régionale de la France dans le Pacifique océanien, une diplomatie multimodale », Journal de la Société des Océanistes [En ligne], 140 I janvier-juin 2015, mis en ligne le 06 juillet 2015, consulté le 02 mai 2019. URL : http://journals.openedition.org/jso/7282 ; DOI : 10.4000/ jso.7282 


\title{
L'intégration régionale de la France dans le Pacifique océanien, une diplomatie multimodale
}

par

\author{
Christian LECHERVY*
}

\section{RÉSUMÉ}

En sadaptant à un espace Pacifique en profonde mutation (États insulaires courtisés par les puissances émergentes, réordonnancé dans de nouveaux sous-ensembles), la France adopte une diplomatie modulaire. Celle-ci se bâtit au travers de son rôle de puissance globale, des liens établis avec l'Union européenne, des particularismes de ses territoires océaniens et de sa stratégie asiatique. Dans les institutions régionales, elle se fonde sur des statuts juridiques multiples et une volonté d'y associer de plein droit la Nouvelle-Calédonie, la Polynésie française, Wallis-et-Futuna. Elle doit compter avec une architecture régionale articulée autour du Forum des îles du Pacifique et privilégiant les États souverains insulaires. Longtemps étatisées, ces politiques doivent dorénavant faire place, pour répondre aux défis globaux, à nombre d'acteurs non gouvernementaux.

MoTS-CLÉs : collectivités territoriales, diplomatie française, Forum des îles du Pacifique, intégration économique, organisations régionales

\begin{abstract}
By adapting itself to a Pacific region in deep evolution (Island States courted by the emergent powers, reorganized in new sub-institutions), France adopts a modular diplomacy. This one builds through its role as a global power, its links developed with the European Union, the senses of identity of its South Pacific territories and its Asian strategy. In the regional institutions, it bases itself on multiple legal statutes and will to associate it by full memberships New Caledonia, French Polynesia, Wallis and Futuna. It has to count with the regional architecture articulated around the Pacific Islands Forum that supports the sovereign island States. Long established stated policies have now to give way to numerous civil societies and NGO in order to face global challenges.
\end{abstract}

KeYwords: Overseas countries and territories, French diplomacy, Pacific Islands Forum, Economic intégration, Regional organizations
À Nouméa devant les chefs d'État et de gouvernement réunis au siège de la Communauté du Pacifique (CPS), le président de la République François Hollande a réaffirmé le 17 novembre 2014 que la France est un pays du Pacifique et de l'Océanie. Si nombre de Français tiennent pour acquis cette réalité géopolitique, la « Françocéanie »

* Ambassadeur, représentant permanent de la France auprès de la communauté Pacifique et du PROE, christian.lechervy@ diplomatie.gouv.fr

NDLR. - Les analyses et commentaires présentés ici n’engagent que leur auteur.

1. Nous utilisons ce néologisme pour qualifier au temps présent un espace géographique et politique singulier du Pacifique-océanien. Le terme de "Françocéanie " et son dérivé " Françocéanité " veulent exprimer une singularité française dans le Pacifique qui vaut aux territoires ultramarins de la République d’être ni pleinement intégrés à leur région, ni totalement exclus. Il s'agit aussi de qualifier une entité ayant une même langue en partage, une même consubstantialité pour beaucoup de partenaires étrangers - l'Union européenne a classifié la Nouvelle-Calédonie, la Polynésie française, Wallis-et-Futuna au sein du même groupe des Ртом (Pays territoires d'outre-mer) - et bâtissant des stratégies additives à celles de l'État français. Bref, il s’agit d'un sous-ensemble géostratégique étant quelque chose de plus que la France, disposant d'identités multiples et d'autonomies croissantes, nées de la loi-cadre Defferre de 1957 jusqu’au partage de souveraineté inscrit aujourd'hui dans les lois organiques. C'est pourquoi pour qualifier l'espace politico-territorial français d'aujourd'hui, il n'était pas possible de reprendre l'acception de la "France australe ", aussi datée que le journal calédonien homonyme qui s'est voulu de 1889 à 1979 l'organe des intérêts 
ne va pas naturellement de soi. Nombre de pays ignorent que la France demeure possessionnée dans la région. D'autres contestent diplomatiquement cette donne et l'espère temporaire. Ce sont d'ailleurs du cœur même du Pacifique qu'ont été parrainées les résolutions des Nations unies inscrivant successivement sur la liste onusienne des territoires non autonomes la Nouvelle-Calédonie, en décembre 1986, par les îles Salomon, Papouasie Nouvelle-Guinée, Vanuatu², puis la Polynésie française, en mai 2013, par Nauru, Samoa, Salomon, Timor oriental, Tuvalu ${ }^{3}$. En conséquence, en étant à la fois, une puissance administrante et un État ayant une diplomatie globale, la France définit une politique extérieure tournée vers les États territoires du Pacifique qui est à la fois hexagonale et fondée sur sa "Françocéanité ». La diplomatie française s'exécute vers le Pacifique, sur et depuis le Pacifique (Dobell, 2013: 1). En vertu de cette multipolarité, la France prend en compte dans sa diplomatie multilatérale les organisations internationales associant, parmi d'autres, les Etats du Pacifique océanien et celles qui ont pour acteurs principaux les États souverains et les territoires non autonomes du Pacifique. Parallèlement, elle affirme ses ambitions et la défense de ses intérêts dans des institutions multi-régionales, transPacifique et intra-Pacifique. Enfin, la diplomatie française interagit avec un nombre croissant d'acteurs à l'échelle sous-régionale, avec plus de partenaires périphériques $\mathrm{du}$ bassin Pacifique et des collectivités territoriales calédoniennes et polynésiennes plus agissantes. Ces dernières s'ouvrent en outre concomitamment sur et à leur région. Le temps où les États et territoires insulaires n'accordaient de l'attention qu'à un tout petit nombre de partenaires est de surcroît dépassé ce qui a pour conséquence une volonté de réorganiser les architectures transnationales de coopération.

\section{La France face à la pluralité des organisations du Pacifique océanien}

$\mathrm{Au}$ fil des décennies et des évolutions des lois cadres régissant les pouvoirs dévolus à la NouvelleCalédonie et à la Polynésie française ${ }^{4}$, il a fallu à la France conduire une diplomatie "Pacifique " multimodale, intégrant dans sa manœuvre les capacités d'action des gouvernements de Nouméa et de Papeete, sans même parler d'une Union européenne ayant enserré le Pacifique dans ses propres instruments d'action extérieure géographisés (ACP, PTOM, UE-Océanie) $)^{5}$ Bien que ces contraintes internes soient tout à fait dimensionnantes pour la diplomatie de la République, c'est d'abord aux évolutions de l'espace Pacifique auxquelles la France dut s'adapter. La France eut toujours à articuler sa politique vis-à-vis du Pacifique avec son action diplomatique en Asie car le Pacifique océanien ne fut jamais ni hors du temps des rivalités stratégiques, ni hermétiquement isolé du reste du monde.

\section{Une politique océanienne " asianisée »}

Dès la fin de la Seconde Guerre mondiale, la politique conduite par la France dans le Pacifique et au travers de ses territoires du Pacifique océanien a interféré avec son action en Asie du Nord-Est et du Sud-Est. Après avoir cherché à se prémunir des effets des développements du communisme indochinois, il s'est agi de faire face à l'émergence de la République populaire de Chine (RPC) sur la scène politique polynésienne (MohamedGaillard, 2010: 171-197). Cette interaction entre les dynamiques politiques océaniennes et asiatiques a également pesé au fil des décennies sur les principaux partenaires occidentaux de la région, formatant ainsi un peu plus l'asianisation du regard français tourné vers le Pacifique.

Les Australiens n'ont accepté une réinsertion du Japon dans l'espace régional qu'au prix de la mise sur pied de mécanismes de sécurité collective avec les États-Unis et la Nouvelle-Zélande. L'encouragement du sens communautaire océanien n'eut pas d'autres buts pendant longtemps que de tenir la région du Pacifique Sud à l'écart de toute influence périphérique et d'actions subversives venues d'Asie du SudEst, notamment d'Indochine et d'Indonésie.

français dans le Pacifique. Il n’était pas non plus possible de faire notre le néologisme de " Franconésie » (Chesneaux-Maclellan, 1992, 112-118) qui laisse à penser que les territoires océaniens d'aujourd'hui seraient toujours intégralement subordonnés aux intérêts de l'État français et ses relais locaux. Ceteris paribus et compte tenu des particularismes politiques qui sont aussi ceux d'autres pays de la région, on serait fondé à parler également d'une " américanocéanité " pour qualifier l'espace politique pluriel des États-Unis dans la région (territoires annexés ou dépendants, Guam, Hawaìi, Mariannes du nord, Samoa américaines ; territoires en libre association, États fédérés de Micronésie, îles Marshall, Palaos), voire même d'une "zélandocéanité " pour dépeindre les relations en rhizome qui lient la Nouvelle-Zélande à Tokelau ou encore aux îles Cook et Niue. Même si ces néologismes ne rendent pas pleinement compte des spécificités de chaque territoire, ils rappellent la nécessité de ne pas résumer les territoires insulaires non souverains aujourd'hui à leurs seules identités locales ou à leurs puissances tutélaires.

2. Ces trois États proposèrent au Forum des îles du Pacifique une résolution demandant la réinscription de la NouvelleCalédonie à l'ordre du jour du Comité de décolonisation des Nations unies. Cette proposition reçut le 8 août 1986 un assentiment unanime comme en témoigne le communiqué final du Forum des îles du Pacifique (FIP).

3. Le 2 septembre 2012, les États fédérés de Micronésie, à Fidji, les îles Marshall, Kiribati, Nauru, les îles Salomon, le Timor oriental, Tonga, Tuvalu et le Vanuatu ont adopté une résolution appuyant la réinscription.

4. Dans le cas de Wallis-et-Futuna, le maintien du statut de 1961 n’a pas fait évoluer les compétences du territoire en matière de relations internationales.

5. Lors $\mathrm{du} 45^{\mathrm{e}}$ sommet du Forum des îles du Pacifique en août 2014, la haute représentante, Mme Ashton a annoncé la tenue prochaine d'un tel sommet. 
Les efforts d'aide au développement aux États devenus souverains furent, de la même manière, autant l'expression de solidarités entre pays développés et pays en voie de développement que la conséquence des politiques visant à éviter que les États océaniens soient tentés d'aller rechercher un appui diplomatique et des moyens financiers auprès de l'URss et de la RPC. C'est donc bien avant même que les puissances asiatiques ne deviennent le moteur de l'économie mondiale que la politique océanienne de la France a dû s'articuler avec sa diplomatie extrême-orientale, aseanienne et indochinoise.

À l'heure où les enjeux commerciaux sont devenus primordiaux, il s'agit dorénavant de savoir si les territoires insulaires français du Pacifique, voire l'Australie, peuvent être pour la France autant de têtes de pont vers l'Asie en croissance. Historiquement, ce ne sont pourtant pas les intérêts économiques qui poussèrent à penser l'espace en termes "Asie-Pacifique" (AsPac). Ce sont des impératifs de sécurité qui imposèrent à Paris de concevoir sa stratégie à l'échelle d'un théâtre "AsPac" (ministère de la Défense, 2013). Alors que la reconquête du Pacifique fut conduite du Sud vers le Nord, d'Océanie vers l'Extrême-Orient en passant par l'Asie du Sud-Est, il s'est agi, à partir de la fin des années 1940 et cela pendant quarante ans, de faire obstacle à tout risque glissant du Nord-Est de l'Asie vers le Pacifique Sud. Tout en globalisant les défis auxquels l'AsPac devait faire face - même la signature le 8 septembre 1954 à Manille du traité de défense collective pour l'Asie du SudEst qui donna naissance à l'OTASE, fut entendue par la France comme couvrant explicitement la sécurité des établissements français d'Océanie, la Nouvelle-Calédonie et les NouvellesHébrides -, le théâtre stratégique AsPac s'est compartimenté au fil des conflits armés. Des espaces politiques "homogènes " ont même fait leur apparition, nourrissant l'émergence de nouveaux micro-régionalismes (ASEAN; Triple alliance indochinoise; partenariats stratégiques américain avec le Japon, les Républiques de Chine et de Corée ; Pacifique Sud sous leadership australo-néo-zélandais). Certains de ces microrégionalismes institutionnalisés sont demeurés à l'état de projets comme l'Union bouddhiste, d'autres ont pris rapidement le chemin d'un régionalisme-ethnicisé - par exemple le MAPHILINDO $^{6}$. Aujourd'hui, des manifestations identitaires d'entre soi qui ne sont plus propres aux micro-régionalismes des territoires à l'est de l'Inde et au sud de la Chine. Elles semblent tentantes jusque chez nombre d'Océaniens comme en témoignent la consolidation des espaces politiques micronésien, mélanésien et polynésien, quitte à ce que certains États jouent d'identités multiples pour valoriser leur importance politique et stratégique à l'instar de Fidji ou encore l'Indonésie.

Paradoxalement, à l'heure où l'Océanie se cardinalise avec le reste du monde, elle revivifie ses identités particulières. Tout en bâtissant des partenariats structurés avec les puissances émergentes (par exemple, Chine, Corée, Inde, Turquie), les territoires océaniens trouvent utiles, indispensables même de se retrouver entre eux. Non seulement à l'échelle insulaire, sans les Australiens voire les Néo-Zélandais ( $c f$. les appels fidjiens au retrait de Canberra et de Wellington du Forum des îles du Pacifique [FIP]), mais également entre Micronésiens, Mélanésiens ( $c f$. Groupe du Fer de lance mélanésien [GFLM] et Polynésiens [Groupe des dirigeants polynésiens]. Ces constructions s'élaborent au nom d'une conceptualisation géographique pour partie artificielle mais elles n’en font pas moins sens aujourd'hui dans le champ politique. L'Indonésie vante ainsi sa " mélanésité " pour s'associer au GFLM et contrecarrer les efforts de légitimation internationale des indépendantistes papous, relayés notamment par le Vanuatu ou encore le FLNKS.

En cherchant à mieux imbriquer aujourd'hui sa politique océanienne avec sa politique d'influence en Asie du Sud-Est et en Extrême-Orient, la France - parfois sans en avoir pleinement conscience - met en œuvre une stratégie "AsPac " déjà fort ancienne. Le seul changement radical à l'intérieur de l'espace est d'avoir vu disparaittre une barrière défensive intra-Pacifique. L'extinction de

6. La confédération Maphilindo (Malaisie, Philippines, Indonésie) fut établie en 1963 pour rassembler dans un même espace politique les populations malaises d'Asie du Sud-Est. Elle échoua du fait des différends territoriaux opposant la Malaisie à l'Indonésie et aux Philippines. On se souviendra que Jules Dumont d'Urville proposa à la Société de géographie de Paris en 1831 de dénommer ce même espace territorial Malaisie. Il constituait à ses yeux une des quatre composantes de l'Océanie avec la Micronésie, la Mélanésie et la Polynésie. Par convention, on parlera ici de Malaisie pour désigner l'entité politique antérieure à 1963 (Malaya) et de Malaysie (Malaysia) pour la période postérieure.

7. La politique océano-Pacifique de Jakarta présente plusieurs facettes. Devenue partenaire du dialogue post-Forum des îles du Pacifique depuis 2001, l'Indonésie se présente comme un " pont » entre l'Asie du Sud-Est et le Pacifique insulaire. À ce titre, elle a instauré en 2002 un dialogue du Pacifique du Sud-Ouest avec les Philippines, la Papouasie Nouvelle-Guinée, le Timor-Oriental, l'Australie et la Nouvelle-Zélande. Elle se veut aussi un " partenaire de la sécurité environnementale ". En 2009 en marge de la Conférence mondiale des océans (woc), elle a donc pris l'initiative d'un Triangle corallien pour la sécurité alimentaire, les pêches et les récifs (CTI) avec la Malaysie, les Philippines, la Papouasie Nouvelle-Guinée et les îles Salomon. Ayant obtenu le statut d'observateur au GFLM en 2011, l'Indonésie vante dans cette enceinte son "insularité ", insiste sur le fait qu' " historiquement elle a plus à voir avec les pays du Pacifique qu'avec l'Asie " (Suryodipuro, 2014) et sur son héritage mélanésien. Le paragraphe 3 de la déclaration en neuf points signée à Jakarta en janvier 2014 par le ministre des Affaires étrangères du président Yudhoyono et ses homologues du GFLM souligne, par exemple, le rôle des Indonésiens de culture mélanésienne dans le dialogue avec le GFLM (Joint Statement, 2014). 
la menace communiste et les fantasmes d'appétits territoriaux qui lui ont été prêtés ont pour conséquence une plus grande fluidité des relations économiques et politiques à l'échelle du bassin, sans même parler des mouvements migratoires. Les économies océaniennes s' «asianisent». Les échanges politiques s'institutionnalisent. États et territoires insulaires bâtissent des «Look North Policies » ce qui revient aussi à dire qu'ils regardent plus que jamais vers l'Ouest. Une nouvelle donne stratégique qui laisse à penser qu'une nouvelle ère politique s'ouvre, que l'unipolarité américaine s'estompe et un nouveau "Grand jeu " s'ordonne. Certains comptent même sur une présence française durable pour participer à un endiguement de la montée en puissance chinoise dans le Pacifique (Fisher, 2013 : 272). Toutefois, la transformation de l'espace politico-stratégique a pour conséquence de nourrir un sentiment d'effacement stratégique de la France et de ses alliés tout en rendant plus prégnante la nécessité d'élaborer un nouveau narratif politique, de nouvelles alliances et modes d'insertion régionale. Afin de montrer qu'elle s'inscrit dans un nouveau grand dessein, la France abandonne peu à peu dans son vocabulaire politico-stratégique toute mention au Pacifique Sud pour privilégier une terminologie faisant référence à l' "Asie-Pacifique " ou au "Pacifique». Le premier référentiel a pour avantage de souligner des intérêts stratégiques allant bien au-delà des territoires océaniens possessionnés. Ce changement sémantique rappelle aussi combien la France peine à fixer son langage pour définir la région et ses sousensembles (Mrgudovic, 2008 : 5-10).

\section{Limportance croissante du bi-multilatéralisme}

Aujourd'hui, la France doit compter avec un réseau d'États insulaires ayant un nombre grandissant de partenaires de par le monde. Certains bien quéloignés du bassin Pacifique ont même institutionnalisé leurs échanges sous la forme de sommets avec tous les États océaniens (Cuba, Inde). Néanmoins, c'est la "jeunesse" des États ${ }^{8}$ qui explique la lente progression de leur tissu relationnel et l'attrait qu'ils représentent collectivement dans les enceintes multilatérales ${ }^{9}$ (Guilbert, 2015).

Les États insulaires sont courtisés comme jamais mais nombre des relations récemment bâties sont des partenariats dissymétriques. Les pays extraocéaniens en tirent des avantages immédiats plus importants que les États insulaires. Il est vrai que les capacités des Océaniens à répondre à toutes les sollicitations demeurent limitées, faute de ressources humaines et d'ambassades. Dans ce contexte, les représentations permanentes auprès des Nations unies à New York sont le lieu privilégié de toutes les batailles d'influence. Bien plus que dans les capitales océaniennes, c'est au siège de l'ONu que sont mises en œuvre les diplomaties des États du Pacifique. La France, membre permanent du Conseil de sécurité, y est en résonance depuis longtemps mais elle doit aussi compter avec une dynamique trans-Pacifique densifiée par un nombre croissant de dialogues Océanie+ $1^{10}$ (Chine, Corée, Japon, Taïwan, Thaïlande). Ce relationnel est d'abord le fruit d'initiatives des puissances émergentes. Pour affirmer urbi et orbi leur nouveau statut et leurs ambitions mondialisées, elles veillent à disposer de forums dédiés à chaque région du monde (par exemple Afrique, Asie du Sud-Est, Amérique latine). Selon cette logique de cardinalisation, l'Océanie ne saurait manquer à l'appel. De surcroit, au-delà des rivalités d'influence affichées (par exemple Chine-Japon, Chine-Taïwan), les pratiques diplomatiques sont bien souvent empreintes de mimétismes. Elles ont accéléré la mise sur pied de tels forums, la plupart ayant trouvé leurs marques dans la décennie 1997-2007.

Si les États océaniens se prêtent aisément à ces exercices de bi-multilaréalisation alors qu'ils n'en sont pas les initiateurs, c'est parce qu'ils espèrent être plus écoutés de la communauté internationale. Il est implicitement entendu que le partenaire, quel qu'il soit et où qu'il se trouve, se doive de relayer les préoccupations océaniennes " vitales ", jugées peu portées aujourd'hui par les partenaires historiques de la région (par exemple Australie, États-Unis).

Les États océaniens ne sont pas des États "passifs». Ils ont des intérêts essentiels et entendent les défendre. Pour cela, ils ont besoin de partenaires disponibles dans le temps et ayant des leviers d'influence. Pour autant, les États insulaires ont parfaitement conscience de leur statut de micro-États mais ils n'en veulent pas moins voir leur "pacificité " mieux reconnue. Signe de leur importance et de leur capacité à faire entendre leur identité propre, en 2011 à New York le groupe de pays les accueillant aux Nations unies a été dénommé "Asie-Pacifique " et non plus "Asie». En outre, les États océaniens ne sauraient voir leur parole véhiculée seulement par leurs puissants voisins australiens et/ou néo-zélandais, aux intérêts souvent si différents. Il leur faut donc trouver leurs propres démultiplicateurs d'influence.

Les puissances émergentes qui se montrent disponibles à bâtir une architecture Océanie

8. En moyenne, les États du Pacifique insulaire sont indépendants depuis seulement 36 ans.

9. Les États océaniens représentent $6,7 \%$ des voix à l'Assemblée générale des Nations unies.

10. On désignera par cet axiome toute réunion internationale rassemblant les États et territoires insulaires du Pacifique et une puissance tierce, quelle que soit la géolocalisation de celle-ci. 
+1 sont d'autant plus rassembleuses qu'elles prennent généralement à leur charge pour les rencontres internationales les coûts de transport prohibitifs des délégations. Elles veillent par ailleurs, la Chine communiste en tête, à offrir aux organisations régionales des infrastructures de travail nouvelles (par exemple les aides au bureau du Forum des îles du Pacifique à Pékin ${ }^{11}$, ou à la construction du siège à Port Vila du GFLM). De plus, à l'occasion de ces manifestations les puissances invitantes prennent le plus grand soin à annoncer de nouveaux engagements sonnant et trébuchant (Chien-peng, 2010: 98-110 ${ }^{12}$ ). Les puissances émergentes le font de manière d'autant plus ostentatoire qu'elles n'ont pas de place statutaire dans les organisations régionales anciennes ou plus récentes du Pacifique-océanien, à l'exception du très formel dialogue post-Forum des îles du Pacifique. Même le plus généreux des donateurs et le plus soucieux à user des canaux multilatéraux pour distribuer son aide aux politiques de développement, le Japon, n'a pas de voix décisionnelle dans les organisations sous régionales qu'il finance - comme la Communauté du Pacifique (CPS), ou encore le Programme régional océanien de l'environnement (PROE). Une situation qui est d'ailleurs également celle de l'Union européenne mais qui ne pourra pas durablement rester inchangée. Un défi politique, financier mais aussi juridique car il implique de modifier les traités fondateurs des organisations océaniennes - notamment le traité de Canberra constitutif de la CPS. En attendant, la bi-multilatéralisation des relations du Pacifique océanien avec le reste du monde va bon train.

Comme d'autres puissances moyennes, la France s'est employée à bi-multilatéraliser ses relations avec le monde océanien. Cette méthode lui permet de rayonner mais aussi de s'assurer que ses trois territoires du Pacifique sont associés étroitement à la construction du dialogue régional et sont intégrés de la même manière aux institutions régionales. C'est ainsi que les trois sommets (2003, 2006, 2009) se sont déroulés successivement à Papeete, Paris et Nouméa. Toutefois, on relèvera que ce sont les élus de Nouvelle-Calédonie (par exemple P. Gomès ou P. Germain)) qui se montrent aujourd'hui les plus vindicatifs à demander l'organisation à Paris d'un quatrième sommet France-Océanie et cela dès la fin de l'année 2015 (Question au gouvernement le 15 janvier 2015, déclaration de politique générale du 13 avril 2015 (Germain, 2015 : 13). Il s'agit de fixer autant que faire se peut des rendez-vous réguliers, les rencontres ayant été prévues pour se tenir tous les trois ans.

\section{Une cardinalisation institutionnalisée et cadencée}

Le rythme triennal des rencontres semble convenir aux États océaniens puisque plusieurs de leurs partenaires l'ont fait leur. Le Japon l'a instauré dès 1997. Depuis, les sept rencontres avec les leaders du Pacifique (PALM) ont permis à Tokyo un accès garanti à des ressources halieutiques, de mieux sécuriser ses liaisons maritimes et de s'assurer du soutien de la plupart des États océaniens à sa campagne pour un siège de membre permanent au Conseil de sécurité des Nations unies.

Les rencontres nippo-océaniennes ne sont pas seulement des échanges inter-étatiques formalisés (préparation par des réunions de hauts fonctionnaires (SOM), réunions des ministres...), elles associent également les acteurs transnationaux (par exemple Secrétaire général du FIP, directeur général du PROE...). Des réunions si inclusives que se pose la question d'y associer d'autres partenaires de la scène internationale. La France par exemple a manifesté publiquement un tel intérêt. Le Japon ne l'a pas écarté comme en ont témoigné le communiqué conjoint F. Hollande-S. Abe du 7 juin $2013^{13}$

11. Le second des sommets des leaders du Pacifique avec le Japon (palm 2) fut l'occasion d'annoncer un Good Will Trust Fund d'une valeur de 10 millions de dollars pour financer des projets dans les domaines de l'environnement, de l'énergie et du tourisme. En réponse, la Chine instaura la même année un Fonds pour la coopération avec le Forum des îles du Pacifique. Ce dernier a financé en 2002 pour un million de dollars l'ouverture d'un bureau commercial du fip à Pékin.

12. En avril 2006, par exemple, le premier ministre chinois Wen Jiabao a annoncé lors du premier forum pour le développement économique et la coopération Chine-Pays insulaire du Pacifique (CPIC Forum) qui se tenait à Suva, un Plan d'action pour le développement économique et la coopération, 375 millions de prêts à des taux préférentiels, la création d'un fonds pour faciliter les investissements chinois dans la sous-région, 2000 bourses de formations et un million de renminbis pour lutter contre la malaria. Un mois plus tard, lors du $4^{\mathrm{e}}$ sommet des leaders du Pacifique avec le Japon (PALM 4) à Okinawa, Tokyo précisait que son aide au développement aux pays insulaires du Pacifique allait passer de 279 millions de dollars par an à 357 millions. En septembre 2006, c’était au tour du président taïwanais Chen Shui-bian de s'engager à développer la coopération économique dans les domaines de l'agriculture, les énergies renouvelables, le tourisme et d'octroyer 100 millions de dollars aux Salomon lors du premier sommet Taïwan-Alliés du Pacifique à Palaos (Chen-yi, 2010 : 121-126). En novembre 2013, lors du $2^{\mathrm{e}}$ CPIC Forum à Canton, il fut annoncé un milliard de dollars de prêts concessionnels pour des projets d'infrastructure et un milliard supplémentaire pour des prêts commerciaux visant à soutenir le développement économique et social de la région. (http://english.mofcom.gov.cn/article/newsrelease/significantnews/201311/20131100386982.shtml). Lors du sommet japonais triennal qui suivit (Iwaki, 23 mai 2015), le premier ministre nippon a surenchéri et annoncé 55 milliards de yens d'aide au développement pour les trois prochaines années et 4000 bourses (Construire ensemble un futur prospère, 2015 : http://www.mofa.go.jp/a_o/ocn/page4e_000261.html).

13. Le point 1 , paragraphe 5 du communiqué conjoint du 7 juin 2013 précise que les deux pays se reconnaissent mutuellement comme étant du Pacifique et annoncent le renforcement de leur dialogue sur la région, en vue d'une coopération régulière au sein des organisations régionales du Pacifique. Il est également précisé que le Japon « a accueilli positivement 
(Communiqué conjoint entre la France et le Japon, 2013) ou encore l'invitation lancée à la secrétaire d'État au Développement et à la Francophonie Mme A. Girardin pour participer à un side event du sommet d'Iwaki en mai 2015 consacré aux changements climatiques. Ces contacts permettent à la France d'affirmer son rôle de puissance globale, sa "pacificité " et de valoriser le potentiel coopératif de ses territoires du Pacifique océanien.

La France n'est pas la seule puissance périphérique à se voir associer aux mécanismes Océanie+1. L'Australie et la Nouvelle-Zélande ont ainsi été invitées comme observateurs au premier Forum Thaillande-pays insulaires du Pacifique (TPIF, Bangkok, 4-8 août 2014). En conséquence, la France doit-elle chercher à se joindre au plus grande nombre des sommets Océanie +1 ? Si cela fait incontestablement sens avec les pays avec lesquels elle entretient un dialogue politique global ou plus particulier sur les questions du Pacifique, l'exclusion de certains forums n'est pas sans danger pour les intérêts français puisqu'elle marginalise doublement la France, en n'associant pas celle-ci et/ou ses territoires ultramarins aux échanges sur les réponses à apporter aux défis transnationaux de la région mais également en faisant des seuls États et territoires ${ }^{14}$ membres de plein droit du Forum des îles du Pacifique la seule pierre angulaire des dialogues Océanie+1. Une approche que l'on retrouve dans le dialogue qui se met en place avec la Corée du Sud et qui offre au FIP des moyens financiers nouveaux dont il paraît vain d'imaginer qu'ils puissent bénéficier, d'une manière ou d'une autre, aux territoires non membres de plein droit du Forum. Pourtant, Séoul a doté son fonds pour la coopération avec le FIP (RPCF) de moyens importants, un million de dollars par an pour les années 2013-2016. Certes en s'appuyant sur le FIP et son secrétariat de Suva, Bangkok et Séoul qui sont tous deux partenaires du dialogue post-Forum, se facilitent la tâche pour organiser toute rencontre internationale avec les Etats océaniens. Les deux pays asiatiques n'ont quasiment pas de relais diplomatiques dans le Pacifique insulaire pour monter des réunions mobilisant des leaders géographiquement très dispersés et disposant d'une faible logistique administrative. Mais en excluant les territoires CPWF (Nouvelle-Calédonie, Polynésie française, Wallis-et-Futuna) des mécanismes Océanie+1, cela met à mal leur connectivité " Asie-Pacifique " potentielle, rend difficile les interactions susceptibles d'être établies avec les dispositifs mis en place avec l' ${ }^{A P E C}{ }^{15}$, voire leur intégration dans les mécanismes de coopération Sud-Sud. Cela est dommageable dans la mesure où la NouvelleCalédonie et la Polynésie française possèdent des instruments nationaux de coopération scientifique mobilisables et sont capables d'amener de l'extérieur des compétences faisant régionalement défaut. Les territoires CPWF sont notamment à même d'œuvrer efficacement au profit de tierces parties du Pacifique océanien avec les agences spécialisées d'aide au développement coréenne (KOICA) ou thailandaise (TICA) ${ }^{16}$ et de leur apporter leurs propres leviers financiers dans le cas de la Nouvelle-Calédonie et la Polynésie française. On pourrait en dire tout autant avec l'Indonésie, elle aussi membre du dialogue postForum tout comme deux autres pays aseaniens, la Malaysie et les Philippines.

La plupart des pays asiatiques offrent la possibilité de coopérer avec des pays soucieux d'adopter les normes internationales édictées par le Comité d'aide au développement de l'OCDE, autant de règles que d'autres nouveaux donateurs, à commencer par l'Inde et la RPC, se montrent rétifs à mettre en œuvre. Mais en écartant ipso facto la Nouvelle-Calédonie et la Polynésie française de certains forums régionaux, certains donateurs adoptent une attitude discriminante en rupture avec la réflexivité des relations souhaitables à l'échelle du bassin Pacifique même s'ils n'en ont pas forcément pleinement conscience. De leur côté, les territoires français ne font pas de même. Nouméa notamment se montre attentive en particulier sur les sujets relatifs à la lutte contre les changements climatiques, à associer le plus grand nombre de partenaires à ses rencontres. À ce titre, le ministre thaïlandais de l'Intérieur en charge de la lutte contre les désastres naturels a même été invité

l'intérêt exprimé par la France pour les sommets des dirigeants des îles du Pacifique (PaLM)». Dans le communiqué de presse conjoint du 5 mai 2014, il est précisé au point 2.16 des coopérations conjointes pour venir en aide aux États insulaires du Pacifique vulnérables aux effets du changement climatique.

14. Deux des quatorze pays insulaires invités par les Thaïlandais ne sont pas membres des Nations unies, les îles Cook et Niue.

15. En octobre 2013 par exemple, la Corée du Sud au sommet de Bali s'est fait le porte-parole des États insulaires du Pacifique et a proposé qu'ils bénéficient des actions mises en œuvre dans le cadre de l'APEC Climate Center installé à Pusan, or seule la Papouasie Nouvelle-Guinée est membre de l'APEC.

16. Ces deux agences sont relativement récentes. Elles ont été respectivement crées en 1991 et en 2004 . Leur expérience est encore somme toute limitée. Au cours des deux dernières décennies, l'assistance sud-coréenne aux États insulaires du Pacifique s'est élevée à 50 millions de dollars. Dans le cas de la Thaillande, le plus grand nombre de projets a été conduit avec le groupe de pays dit CLMv (Birmanie, Cambodge, Laos, Viêt Nam) même si quelques projets ont été mis en œuvre au-delà de l'Asie du Sud-Est. Au nom de la coopération Nord-Sud-Sud, Bangkok a ainsi mis en œuvre un projet au Sénégal avec la France. 124 opérations de prêt pour un montant de 25,7 milliards de baths ont été conduites. En moyenne, 5 à 10 projets nouveaux sont adoptés par an essentiellement dans les domaines de l'agriculture, les développements communautaires, l'éducation, l'environnement, la pêche, la santé et le tourisme. 
par le président du gouvernement calédonien à se joindre au troisième sommet d'Oceania $21^{17}$ (28-30 avril 2015) alors que le Royaume est sous sanctions européennes.

\section{Une multivectorialisation très politique du Pacifique}

Si avec certains partenaires d'Asie du NordEst voire du Sud-Est il est possible de concevoir un mode d'association de la France à leurs dialogues institutionnels avec l'Océanie, cela paraît bien plus difficile à envisager avec la Chine car l'association de la France mettrait à mal le narratif voulant que les rencontres ChineOcéanie soient des mécanismes de coopération Sud-Sud. Quant à l'association d'entités non souveraines, cela introduirait implicitement, tôt ou tard, la question de Taïwan. Or Taipeh entretient des relations particulièrement étroites avec les États insulaires. Depuis le début de la décennie 70 , la rivalité sino-taïwanaise latente a eu pour conséquence d'enkyster Pékin et Taipeh dans les jeux politiques intérieurs des États océaniens (Lechervy, 2015b) et d'ordonnancer deux mécanismes concurrents d'échanges bimultilatéralisés.

Derrière des manœuvres diplomatiques et géopolitiques se cachent des calculs géoéconomiques. Les dimensions mercantiles des sommets bi-multilatéralisés sont manifestes tout en étant en rien spécifiques au monde chinois. Le Forum Thaïlande-pays insulaires du Pacifique (TPIF) sert les intérêts économiques de Bangkok notamment ses pêcheurs de thon et les entrepreneurs qui cherchent à obtenir des concessions forestières en Papouasie NouvelleGuinée. La Corée du Sud, tout en faisant implicitement obstacle au développement de réseaux d'influence nord-coréens dans la région ${ }^{18}$, cherche à valoriser ses savoir-faire industriels en premier lieu dans le secteur des industries des énergies renouvelables auprès des États insulaires qui veulent réduire au plus vite leur dépendance vis-à-vis des énergies hydrocarbonées fort coûteuses à importer.

Au-delà d'intérêts commerciaux bien compris, le bi-multilatéralisme est aussi très politique. Dans le cas chinois par exemple, il permet d'afficher combien le Japon n'est plus le principal partenaire économique et d'aide au développement de la région. Il s'agit de dévaloriser stratégiquement le Japon en particulier dans le Pacifique Sud, les positions nippones étant plus affirmées en Micronésie où l'influence taïwanaise est aussi plus prégnante. L'agenda politique du bi-multilatéralisme est si évident que dès que Pékin eut instauré son Forum de développement économique Chine-Pays des îles du Pacifique (avril 2006), la République de Chine s'est inquiétée de cette nouvelle caisse de résonance et s'est empressée d'instituer des sommets Taïwan-Alliés du Pacifique (novembre 2006). Au fond, c'est en comprenant que l'Océanie ne voulait pas se trouver enfermée dans les rivalités sino-nippones et sino-taïwanaises que la Corée du Sud et certains pays de l'ASEAN se sont montrés disponibles à l'instauration de nouveaux partenariats. En outre, le bi-multilatéralisme permet d'égrainer un nouveau récit politique des puissances émergentes, aussi valorisant à l'intérieur des frontières que sur les théâtres extérieurs. C'est l'occasion pour les nouveaux Dragons de souligner leurs succès et le chemin qui les a conduit d'États récipiendaires de l'aide internationale à celui de donateurs (Corée du Sud, Singapour) même si dans certains cas (par exemple la Thaillande) les pays sont à la fois des donateurs et des bénéficiaires de l'aide internationale.

La politisation des forums tient également au rôle donné aux ministres des Affaires étrangères et au choix des puissances émergentes de s'adresser au plus politique des forums, le FIP. C'est ainsi que Séoul orchestre régulièrement des réunions de ministres des Affaires étrangères avec les îles du Pacifique (mai 2011, novembre 2014) et la Cité-État de l'ASEAN cherche à s'associer au FIP et au Secrétariat de la Communauté du Pacifique. Cette dynamique de rapprochement n'est pas propre aux pays riverains du bassin Pacifique. L'Inde a organisé un sommet océanien autour du premier ministre N. Modi en déplacement à Fidji (novembre 2014) et a annoncé vouloir réitérer l'événement. De la même manière, une première réunion ministérielle Cuba-îles du Pacifique s'est tenue en septembre 2008 à La Havane. Mais alors que l'Océanie se multi-vectorialise, aucun forum nouveau n'a été constitué avec les ÉtatsUnis, pire Washington a fermé pour des raisons d'arbitrages budgétaires ses représentations aux îles Salomon et aux Samoa en 1993 (Crocombe, 1995, 278). En outre, Washington n'a pas plus cherché à élargir l'APEC vers le Pacifique insulaire au-delà de la Papouasie Nouvelle-Guinée qui accueillera en 2018 le sommet des chefs d'État et de gouvernement de l'organisation transPacifique.

17. Les sommets Oceania 21 se veulent les rencontres océaniennes annuelles du développement durable. Ils rassemblent les représentants des États et territoires insulaires, des responsables coutumiers, associatifs et scientifiques.

18. Fidji est le seul État du Pacifique insulaire à avoir ouvert une représentation diplomatique à Pyongyang. Le Commandore Esala Teleni y a présenté ses lettres de créances en août 2012. Ce geste a été présenté comme l'expression de la "Look North Policy" adoptée par Fidji. Les deux pays entretiennent toutefois des relations diplomatiques depuis 1975. Un arrangement administratif de coopération a été paraphé en marge du sommet des non-alignés en août 2012 à Téhéran. Il couvrirait les relations politiques et commerciales bilatérales. 


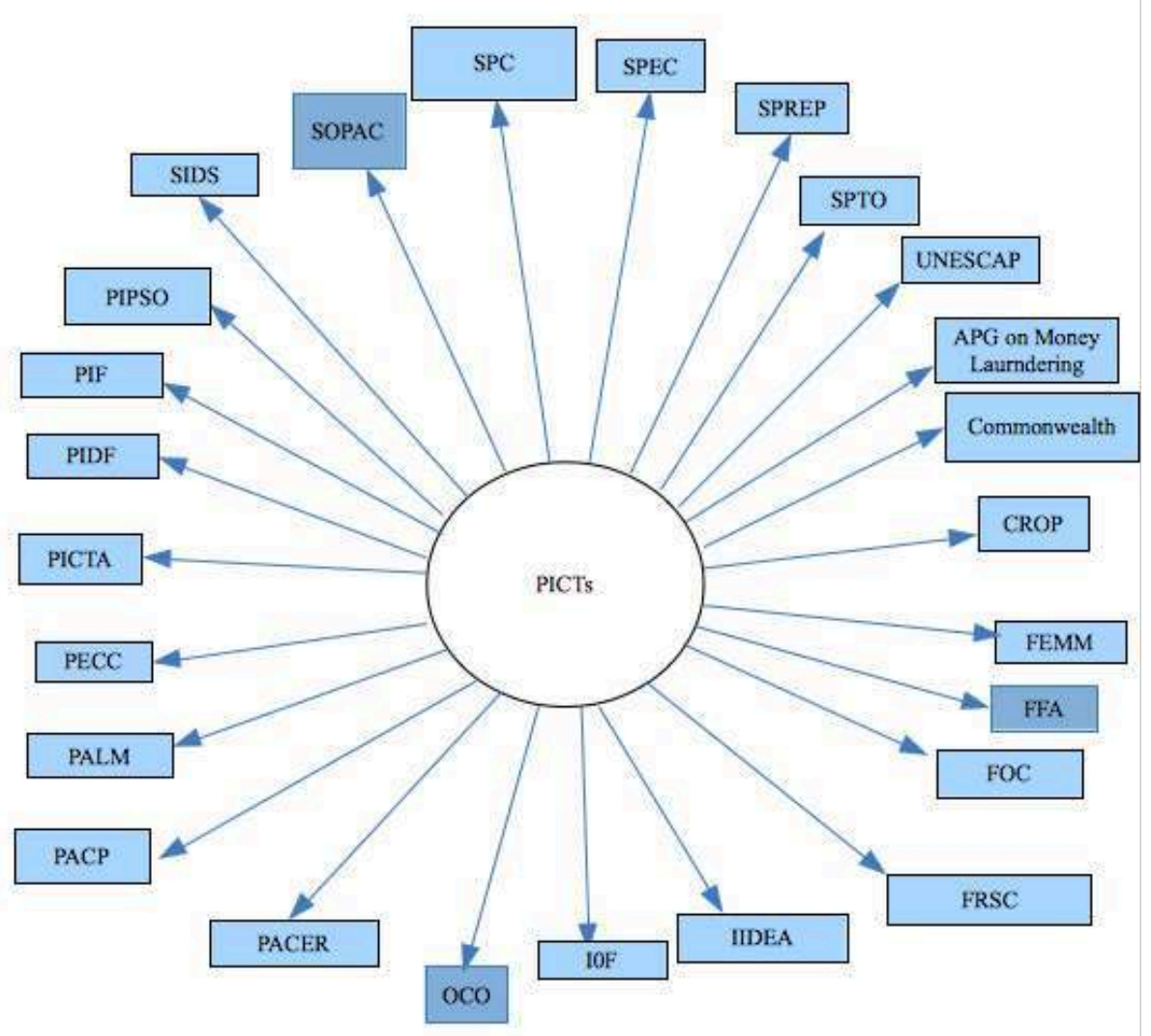

Figure 1. - Les États et territoires insulaires océaniens dans les organisations régionales du Pacifique océanien (C C. Lechervy)

(Voir le tableau 2 ci-dessous pour la signification des abréviations.)
Dans ce contexte, les puissances émergentes asiatiques ont beau jeu de vanter au travers des forums bi-multilatéralisés le siège des institutions internationales qu'elles accueillent (par exemple l'Institut mondial pour une croissance verte ${ }^{19}$, le Fonds vert pour le climat, la Commission économique et sociale pour l'Asie-Pacifique) mais également leurs attraits économiques et leurs savoir-faire. Elles ne sont pas les seules. Les Émirats arabes unis ou Cuba ont développé des coopérations plus techniques avec le Pacifique pour consolider l'assise internationale de certaines de leurs institutions comme l'Agence internationale pour les énergies renouvelables ou l’École latino-américaine de médecine.

\section{Macro-multilatéralisme versus micro-régionalisme}

Ces nouveaux pôles de coopération démontrent que l'espace océanien s'imbrique institutionnellement avec lui-même et le reste du monde ( $c f$. figure 1). Il en est de même pour les organisations sous-régionales. En 2014, l'Assemblée générale des Nations unies a ainsi décidé d'accorder, avec le soutien de la France, un statut d'observateur permanent à la CPS, le FIP en bénéficiant déjà depuis 1994. Ce qui est vrai pour les méta-organisations l'est tout autant pour les États insulaires pris individuellement. Les États du Pacifique océanien s'insèrent, de plus en plus, dans des organisations internationales extrarégionales.

Le macro-multilatéralisme s'accompagne d'une présence renforcée des États océaniens dans plusieurs institutions internationales - par exemple la Banque asiatique de développement ${ }^{20}$, le Mouvement des non-alignés ${ }^{21}$ ou encore l'OMC $^{22}$. Ces réseaux sont efficacement mobilisés (par exemple l'Alliance des petits États insulaires) et se densifient en même temps que se renforce un régio-multilatéralisme trilatéralisé (Sommet des chefs des chefs des pouvoirs exécutifs de Micronésie occidentale, 2003, Groupe du Fer de lance mélanésien, 1988, Groupe des dirigeants polynésiens, 2012). Cette dynamique montre combien le multilatéralisme océanien a aussi besoin de se rétracter sur des identités étroites (Micronésie, Mélanésie, Polynésie) au moment

19. Le Kiribati et la Papouasie Nouvelle-Guinée sont des membres fondateurs du GGgI. En avril 2013, Fidji a rejoint l'organisation dont le siège est à Séoul.

20. Six des treize États et territoires du Pacifique ont rejoint la BAD depuis 1990.

21. Fidji, Papouasie Nouvelle-Guinée, Timor oriental, Vanuatu.

22. Fidji (1996), îles Salomon (1996), Papouasie Nouvelle-Guinée (1996), Samoa (2012), Tonga (2007), Vanuatu (2012). 


\begin{tabular}{|l|c|c|c|c|c|}
\hline Sous-région & $\begin{array}{c}\text { Nombre d'etio } \\
\text { (en\%) }\end{array}$ & $\begin{array}{c}\text { En \% de la } \\
\text { population totale }\end{array}$ & $\begin{array}{c}\text { En \% de la } \\
\text { superficie } \\
\text { terrestre }\end{array}$ & $\begin{array}{c}\text { En \% des zEE } \\
\text { océaniennes }\end{array}$ & $\begin{array}{c}\text { En \% du PIB } \\
\text { total }\end{array}$ \\
\hline Mélanésie & 23 & 89 & 98 & 26,7 & 90,9 \\
\hline Micronésie & 32 & 5 & 0,5 & 38,1 & 3,5 \\
\hline Polynésie & 45 & 6 & 1,5 & 35,2 & 5,6 \\
\hline
\end{tabular}

Tableau 1. - Poids relatifs en Océanie de la Mélanésie, de la Micronésie et de la Polynésie (données 2013) (Sources, CPS Rapport de résultats 2013-2014, ADo 2015 Financing Asia's Future Growth)

même où il institutionnalise ses relations avec le reste du monde. Il sera intéressant de voir si la triangulation fait apparaître une géométrie politique isocèle, donnant à chaque pôle le même poids alors que les déséquilibres en matière de ressources économiques et territoriales sont considérables ( $c f$. Tableau 1). Les cinq pays mélanésiens représentent près de $98 \%$ des terres émergées et des ressources terrestres. Les dix-sept États et territoires insulaires océaniens (ETIO) de Micronésie et de Polynésie ne couvrent même pas à eux seuls toute la superficie terrestre du Vanuatu, d'où l'importance capitale pour eux, plus que pour tout autre, d'accéder et de pouvoir exploiter durablement les ressources hauturières. De même, l'apparition de structures régionales identitaires fera-t-elle naître des coopérations privilégiées entre Mélanésiens et Polynésiens ou encore entre Mélanésiens et Micronésiens ? Si cela devait se vérifier les modes de fonctionnement du Forum des îles du Pacifique en seraient radicalement modifiés.

Les nouvelles dynamiques intra-régionales réévaluent également la place à accorder aux territoires français. En 2013, le PIB de la Polynésie française représentait $74,8 \%$ du monde polynésien. Celui de la Nouvelle-Calédonie constituait $6,2 \%$ du total mélanésien mais il est le deuxième de la sous-région après celui de la Papouasie Nouvelle-Guinée.

Ce qui se joue avec le développement du régio-multilatéralisme, c'est également la verticalisation des organisations régionales et l'équilibre des institutions dédiées au Pacifique océanien surtout à l'heure où les autorités fidjiennes remettent en cause la composition du FIP voire son rôle de pivot régional ( $c f$. figure 2 ).

Fidji se montre le plus offensif sur le sujet mais certains de ses voisins n'en pensent pas moins. En refusant que l'Australie et la Nouvelle-Zélande soient parties prenantes aux institutions du Forum des îles du Pacifique (Poling, 2015), Suva remet en cause l'ordre établi au lendemain de la Seconde Guerre mondiale ou tout au moins depuis le début des années 70 lors de la mise sur pied du FIP. En ébranlant le Forum, c'est toute l'architecture régionale qui est remise en cause ( $c f$. figure 2 ).

Pour autant, le réordonnancement de l'architecture régionale ne saurait être le fruit des (dés)accords du binôme australo-fidjien. La plupart des défis de la région (climatiques, économiques, migratoires, sanitaires) se moquent bien des statuts juridiques des territoires insulaires. C'est ce que la France s'emploie à répéter à intervalles réguliers pour souligner combien il serait aussi injuste que contre-productif de ne pas associer ses territoires du Pacifique aux actions de coopérations transnationales et aux organisations dont se dote la région. Le système des Nations unies paradoxalement prend mieux en considération cette attente française.

Les programmes multilatéraux d'aide au développement doivent répondre à des défis qui font fi des statuts juridiques des territoires. Les organisations onusiennes installent donc des bureaux à vocation régionale au coeur du Pacifique insulaire et des bureaux de coopération déconcentrés, ce qui facilitent les contacts avec les territoires français, offrant ainsi plus d'occasion d'échanger et co-opérer avec les collectivités ( $c f$. l'ouverture en 2014 d'un bureau à Apia du Programme des Nations unies pour l'environnement, PNUE, et la mise sur pied du Dialogue environnemental Asie-Pacifique, SEPD). Le micro-régionalisme a ceci d'avantageux pour la France qu'il estompe la distinction entre les territoires souverains et ceux qui ne le sont pas. Il est plus facile pour la Nouvelle-Calédonie ou la Polynésie française d'y trouver place y compris dans des organisations sous-régionales plus politiques. Personne ne discute la vocation, à plus ou moins long terme, de Nouméa à se joindre au Groupe du Fer de lance mélanésien ou encore à Papeete d'être partie prenante du Triangle polynésien. Si la Polynésie française n'a pu convaincre récemment ses voisins de lui confier le siège d'un secrétariat du Groupe des dirigeants polynésiens, cette décision n'a rien à voir avec son degré d'émancipation vis-à-vis de la République française. Le désaccord portait uniquement sur l'utilité d'une structure de soutien, son coût et sur l'appréciation portée sur le leader attaché à ce projet, bien plus que sur le caractère indépendant ou non du territoire. Quant à la place future de la Nouvelle-Calédonie au GFLM, elle est liée intimement à l'avenir institutionnel et politique du territoire. 




FIgURE 2. - Verticalisation des organisations régionales du Pacifique océanien (C) C. Lecherv)

La tyrannie des distances, la volonté des États d'accueillir sur leur sol des organisations internationales créatrices d'emplois nationaux de soutien ${ }^{23}$ et la rivalité entre les institutions favorisent le micro-régionalisme. Ce dernier, bien plus que les méta-organisations, fait place à des experts et des bénéficiaires de Nouvelle-Calédonie, de Polynésie française, de Wallis-et-Futuna. Toutefois si cette déconcentration des centres de décision opérationnelle permet de mieux associer la Françoéanie au quotidien, encore faut-il à cette dernière ainsi quà la France de s'adapter à un monde intra-Pacifique en pleine recomposition.

Alors qu'au cours de la dernière décennie le nombre d'organisations régionales a été réduit de 16 à 10, la rationalisation passée ne fait pas obstacle à l'émergence de nouvelles structures transnationales d'échanges et de coopération se rassemblant sur une base annuelle (comme le Forum de développement des îles Pacifique, FDIP, 2012, ou le Sommet Oceania 21 du développement durable, 2013). La multiplication du nombre des forums mobilise les énergies et les ressources mais complexifie d'autant l'action diplomatique non seulement de la France, de ses partenaires mais également de la NouvelleCalédonie, de la Polynésie française voire de Wallis-et-Futuna. Non seulement le nombre de forums régionaux croît mais au sein de ceuxci on assiste à une représentation plurielle de la France, en nombre (1 à 4) et en variété de statuts (associé, exclu, membre de plein droit, observateur). À la complexité de la "pacificité " s'ajoute l'«asianisation" du bassin Pacifique, l'insertion non uniforme des insulaires dans les organisations de la mondialisation et une « Françocéanité » toujours plus complexe.

\section{Une France plurielle dans les organisations du Pacifique océanien}

Dans les enceintes internationales d'"AsiePacifique ", la France agit sous divers statuts tout comme ses trois collectivités d'outre-mer. On peut classer les modes d'association aux organisations transnationales AsPac en huit grandes catégories,

1) La France représente seule juridiquement ses territoires océaniens comme le Comité régional 


\begin{tabular}{|l|l|}
\hline $\begin{array}{l}\text { ACPIP } \\
\text { (PICTA) }\end{array}$ & $\begin{array}{l}\text { Accord commercial entre les pays insulaires } \\
\text { du Pacifique }\end{array}$ \\
\hline APF (FFA) & Agence des pêches du Forum \\
\hline $\begin{array}{l}\text { APREPE } \\
\text { (PACER) }\end{array}$ & $\begin{array}{l}\text { Accord du Pacifique sur des relations } \\
\text { économiques plus étroites }\end{array}$ \\
\hline BAD (ADB) & Banque asiatique de développement \\
\hline BCIIP (PT\&I) & $\begin{array}{l}\text { Bureau pour le commerce et les } \\
\text { investissements des îles du Pacifique }\end{array}$ \\
\hline BM (WB) & Banque mondiale \\
\hline CCEP (PECC) & Conseil de coopération économique du Pacifique \\
\hline $\begin{array}{l}\text { CCTFP } \\
\text { (PFTAC) }\end{array}$ & $\begin{array}{l}\text { Centre pour la coopération technique et } \\
\text { financière dans le Pacifique }\end{array}$ \\
\hline $\begin{array}{l}\text { CESAP } \\
\text { (UN - ESCAP) }\end{array}$ & $\begin{array}{l}\text { Commission économique et sociale des } \\
\text { Nations Unies pour l'Asie - Pacifique }\end{array}$ \\
\hline $\begin{array}{l}\text { CGAPC } \\
\text { (SOPAC) }\end{array}$ & $\begin{array}{l}\text { Commission des géosciences appliquées du } \\
\text { Pacifique Sud }\end{array}$ \\
\hline COEP (SPBEA) & Conseil océanien d'évaluation pédagogique \\
\hline COF (FOC) & Comité des officiels du Forum \\
\hline CORP (CROP) & Conseil régional des organisations du Pacifique \\
\hline CPRF (FRSC) & $\begin{array}{l}\text { Commission des pêches du Pacifique centre- } \\
\text { ouest }\end{array}$ \\
\hline États et territoires insulaires océaniens \\
\hline Pacifique
\end{tabular}

\begin{tabular}{|c|c|}
\hline FIP (PIF) & Forum des îles du Pacifique \\
\hline FDIP (PIDF) & Forum de développement des îles du Pacifique \\
\hline GCM (MCG) & Groupe de contact ministériel \\
\hline GDP (PLG) & Groupe des dirigeants polynésiens \\
\hline GFLM (MSG) & Groupe du Fer de lance mélanésien \\
\hline IIDAE (IIDEA) & $\begin{array}{l}\text { Institut international pour la démocratie et } \\
\text { l'assistance électorale }\end{array}$ \\
\hline ODO & Organisation des douanes océaniennes \\
\hline OIF (IOF) & Organisation internationale de la francophonie \\
\hline OMC (WTO) & Organisation mondiale du commerce \\
\hline OMI (IOM) & Organisation maritime internationale \\
\hline OSPIP (PIPSO) & $\begin{array}{l}\text { Organisation du secteur privé des îles du } \\
\text { Pacifique }\end{array}$ \\
\hline OTPS (SPTO) & Organisation du tourisme du Pacifique Sud \\
\hline PACP (PACP) & $\begin{array}{l}\text { États du Pacifique du groupe Afrique } \\
\text { Caraïbes Pacifique }\end{array}$ \\
\hline PEID (SIDS) & Petits États insulaires en développement \\
\hline PIDP (PIDP) & $\begin{array}{l}\text { Programme de développement des îles du } \\
\text { Pacifique }\end{array}$ \\
\hline PROE (SPREP) & $\begin{array}{l}\text { Programme régional océanien de } \\
\text { l'environnement }\end{array}$ \\
\hline РTOM (Ост) & Pays et territoire d'outre-mer \\
\hline RDP (PALM) & Rencontre des dirigeants du Pacifique \\
\hline RMEF (FEMM) & Rencontre des ministres économiques du Forum \\
\hline RMCF (FTM) & Rencontre des ministres du Commerce du Forum \\
\hline
\end{tabular}

TABLEAU 2. - Liste des abréviations (et acronymes anglophones entre parenthèses)

de l'Organisation mondiale de la Santé pour le Pacifique occidental à Manille ${ }^{24}$ par exemple.

2) La France représente ses territoires dans des compétences qui leur ont été déjà déléguées, ainsi, bien que membre de l'Organisation Météorologique Mondiale (wMO) depuis le 5 décembre 1949, la Nouvelle-Calédonie est représentée dans cette enceinte par la France qui en est membre tout comme la Polynésie française.

3) La Nouvelle-Calédonie et la Polynésie française sont des partenaires "juniors" (par exemple, la France est un membre fondateur de la Commission économique et sociale Asie-Pacifique des Nations unies, la Nouvelle-Calédonie et la Polynésie française n'en sont que des membres associés depuis 1992).

4) La Nouvelle-Calédonie, la Polynésie française, Wallis-et-Futuna et la République française sont sur le même pied d'égalité comme membres de plein droit (par exemple Communauté du
Pacifique, CPS, Programme régional océanien de l'environnement, PROE).

5) L'État a un statut « dégradé " par rapport à celui accordé à ses territoires océaniens (par exemple au Forum des îles du Pacifique, la France est un partenaire du dialogue post-Forum depuis 1989, la Nouvelle-Calédonie et la Polynésie française sont des membres associés depuis $2006^{25}$, Walliset-Futuna un observateur depuis 2006).

6) La France n'est pas membre mais les collectivités territoriales sont associées (par exemple la Nouvelle-Calédonie bénéficie du statut d'observateur permanent à l'Agence des pêches du Forum, FFA, depuis mai 1998, la Polynésie française et la Nouvelle-Calédonie sont des membres associés depuis 1991 de la Commission des géosciences appliquées du Pacifique, sopaC, la Nouvelle-Calédonie et la Polynésie française sont des membres constitutifs du Programme de développement des îles du Pacifique, PIDP).

24. En février 2012, Harold Martin alors président du gouvernement de Nouvelle-Calédonie a fait part de la volonté de Nouméa de voir son statut évoluer et être rehaussé au niveau de celui accordé à Hong Kong et Macao qui participent à cette instance pour leur propre compte.

25. En 2006, la Nouvelle-Calédonie a fait connaître officiellement son souhait de devenir un membre de plein droit de l'organisation. Le 25 août 2014, le président Gaston Flosse en a fait de même par courrier auprès du secrétaire général du FIP pour le compte de la Polynésie française. Les seules différences entre un membre à part entière et un membre associé résident dans la participation à la retraite des leaders, dans le paiement d'une cotisation minorée et dans l'emploi de ressortissants des membres de plein droit au sein du secrétariat du FIP. 
7) L'État est exclu au profit d'acteurs infraétatiques - la participation du FLNKS au Groupe du fer de lance mélanésien ${ }^{26}$ en est une illustration. 8) Ni l'État, ni les collectivités territoriales ne sont présentes, comme c'est le cas au Conseil océanien d'évaluation pédagogique (SPBEA) ou encore au Centre pour la coopération technique et financière dans le Pacifique (PFTAC).

\section{Une diversité de statuts pénalisante}

La diversité des statuts nuit à la lisibilité de la politique française, à une certaine cohérence d'ensemble mais également à la place qui peut être légitimement octroyée à la Nouvelle-Calédonie et à la Polynésie. Elle rebute l'État-stratège et lui fait mettre de côté souvent l'une ou l'autre des structures auxquelles elle est Partie - par exemple à l'UNESCAP. En outre, le schéma institutionnel déjà bien compliqué ne doit pas faire oublier que l'atoll de Clipperton dans le Pacifique Nord est un cas singulier à garder à l'esprit. Certes, il ne rattache pas par lui-même la France à une organisation (sous) régionale mais le sujet n'est pas anodin. Clipperton, même sans habitant, contribue lui aussi à faire de la France un État possessionné du Pacifique. La République dispose à ce seul titre d'une zEE conséquente, $6,6 \%$ de sa zone économique exclusive du Pacifique et $2 \%$ du total de ses ZEE. À l'échelle européenne, il s'agit d'une superficie comparable à celle d'un pays comme la Suède et à l'échelle mondiale cette zone économique exclusive se range au $56^{\mathrm{e}}$ rang (Lechervy, 2015a). Dès lors, peut-on imaginer la France comme État partie à une organisation régionale du Pacifique au seul titre de ses confins non habités? Une question qui pourrait bien être posée à Londres avant Paris. Si d'aventure, le Royaume-Uni devait évacuer la cinquantaine d'habitants résidant sur les îles Pitcairn, scénario envisagé épisodiquement, qu'adviendrait-il du siège de l'archipel à la CPs par exemple, alors que la Grande-Bretagne s'est déjà retirée de l'organisation en $2005^{27}$ ? Le scénario d'un membre d'une organisation du Pacifique océanien sans habitant sur son sol pourrait bien être prochainement autre chose qu'un cas d'école. L'élévation du niveau de la mer du fait des changements climatiques menace l'existence même de certains États souverains, au point que ceux-ci s'interrogent sur le transferement de leurs populations vers des territoires de pays tiers, cela pourrait donc bien donner sens à l'hypothèse de pays sans habitant membre d'organisations régionales du Pacifique.
Ce maillage des statuts nécessite de s'adapter à chaque instance et de pouvoir ordonnancer une manœuvre d'ensemble. Pour les territoires d'outre-mer cela impose de disposer d'une administration dédiée. Si à Wallis-et-Futuna c'est le cabinet de l'administrateur supérieur, à Papeete et à Nouméa ce sont deux administrations gouvernementales qui ont été mises sur pied. En Nouvelle-Calédonie, un service de coopération régionale et des relations extérieures a été créé en 2001. Armé de douze personnes, il supervise les relations bilatérales, multilatérales et le commerce extérieur du territoire. Tout comme son pendant polynésien bien que de taille plus modeste, il rapporte au président du gouvernement, seul compétent en matière internationale. $\mathrm{La}$ professionnalisation de ces institutions incite les territoires à mieux rayonner mais également à rapporter publiquement de leurs actions notamment auprès des commissions des relations extérieures des assemblées territoriales. Un contrôle démocratique propre aux territoires ultramarins s'esquisse ainsi.

\section{Des stratégies territoriales plus élaborées}

Les nouvelles administrations territoriales en charge des dossiers internationaux, les présidents des gouvernements, les élus sont en phase d'appropriation de leurs compétences internationales. $\mathrm{Si}$, pour l'essentiel, les affaires étrangères relèvent de la compétence de l'État, les lois organiques ont accordé à la NouvelleCalédonie et à la Polynésie française de nombreuses compétences en matière de relations extérieures. Ces responsabilités étant aussi récentes que sujettes à controverses, elles ne sont assumées encore que partiellement, même si les autorités locales ne peuvent prendre des décisions en matière internationale pouvant aller à l'encontre des engagements internationaux de la République. L'existence d'administrations dédiées aux relations internationales et la volonté politique des élus donnent progressivement de la consistance, de la constance, de la cohérence aux démarches entreprises vers les États et territoires voisins.

L'étranger proche étant l'horizon politique immédiat, la Nouvelle-Calédonie se tourne vers la Mélanésie, la Polynésie française vers le Triangle polynésien. Mais pour être recevables, inscrites dans la durée, encore faut-il que ces stratégies puissent être fondées précisément en droit.

26. On notera toutefois que l'accord de libre-échange signé le 29 juillet 2004 entre les États membres du GFLM dispose à l'article 3 qu'un statut d'observateur permanent est accordé au FLNKs jusqu'à ce que la Nouvelle-Calédonie devienne partie à cet accord.

27. Membre fondateur de la CPS, le Royaume-Uni s'est retiré une première fois de l'organisation en 1996 avant de retrouver son siège en 1998 . 
Dans le cas calédonien en vertu de la loi organique du 19 mars 1999 actualisée le 15 novembre 2013, prise pour l'application de l'Accord de Nouméa $^{28}$; selon l'article 28, les autorités de la République peuvent confier au président du gouvernement des pouvoirs pour négocier et signer des accords dans le domaine de compétence de l'État, avec des États ou des organisations internationales du Pacifique. En 2015, c'est ce qui a été fait dans le cadre d'un accord pluriannuel de coopération avec le Vanuatu. On notera de même que les prérogatives prévues aux articles 31 (association aux organisations internationales ${ }^{29}$ ), 32 (représentation auprès des États et territoires du Pacifique) et 33 (signature de conventions de coopération décentralisés avec des collectivités étrangères) sont également globalement mises en œuvre à la différence des articles 29 et 30 .

Selon l'article 29, dans les domaines de compétence de la Nouvelle-Calédonie, qui sont nombreux, le Congrès peut autoriser le gouvernement à négocier des accords avec les Etats et organisations régionales. Les autorités de la République sont alors informées de l'intention de négocier et peuvent confier au président du gouvernement local les pouvoirs pour signer les accords élaborés. Ce champ d'application est encore très largement inexploré tout comme les compétences édictées à l'article 30 qui précisent que le président du gouvernement de la Nouvelle-Calédonie, le cas échéant, les présidents de province, peuvent être associés, au sein de délégations françaises, aux négociations avec l'Union européenne. Ceci incite les provinces à se doter, elles aussi, de structures en charge des questions internationales ${ }^{30}$.

Dans le cas polynésien, la situation est peu différente si ce n'est que la loi organique confère une compétence plus large en matière de consultation de l'assemblée territoriale et une responsabilité géographique élargie au président de la Polynésie française (article 15 de la loi organique du 27 février 2004), ce qui est utile pour le développement des relations avec la Chine, les États-Unis, le Japon voire le Chili, quatre partenaires historiques de Papeete.

Les dispositions existantes permettent aux territoires de développer un large spectre de coopérations bilatérales. Ainsi, des coopérations de solidarité et d'aide au développement "Nord-Sud" ont été mises sur pied avec Fidji, le Vanuatu, les Tonga. Elles sont susceptibles de se diversifier encore géographiquement à l'avenir (par exemple, îles Cook, Papouasie Nouvelle-Guinée, Salomon). Il y a également des coopérations qualifiées d'égal à égal ou "Nord-Nord" avec l'Australie et la NouvelleZélande dans les domaines administratifs, éducatifs, économiques, sportifs, de santé ou encore de transport. Enfin, il y a les coopérations entre les collectivités territoriales françaises ellesmêmes ( $c f$. l'accord particulier entre l'État, la Nouvelle-Calédonie, Wallis-et-Futuna ; l'accord commercial liant depuis 2001 la NouvelleCalédonie et la Polynésie française). Cet écheveau attache les communautés françaises à leur environnement aujourd'hui, et demain plus encore.

Sans préjuger de l'avenir, les dispositifs existant sont évolutifs. Il s'agit de mettre en cohérence les textes avec les pratiques mais également d'aligner, autant que possible, les dispositions les plus récentes à l'ensemble des territoires. Une démarche de cohérence administrative mais qui rencontre bien des réticences politiques notamment en Nouvelle-Calédonie. Dans son souci pratique d'homogénéisation législative et réglementaire, l'État ne peut donc faire fi des particularismes de chacun de ses territoires. Ainsi en 2013, le projet d'actualisation de la loi organique a été jugé excessif dans l'élargissement des compétences relatives à l'ouverture des représentations du territoire hors des ambassades de la République, choix fait à deux reprises par le passé par Papeete pour des bureaux à Pékin et Wellington. Pour l'heure, en vertu d'un accord signé avec le Quai d'Orsay et le ministère des Outre-Mer en 2012, la Nouvelle-Calédonie ne disposera donc de représentants que dans cinq ambassades de France (Australie, Fidji, NouvelleZélande, Papouasie Nouvelle-Guinée, Vanuatu), sous réserve encore de définir les modalités de recrutement de ses agents ${ }^{31}$.

L'État continuant de constater des lacunes dans les dispositifs juridiques de ses pratiques, il voudra les combler car tout vide juridique est susceptible de contentieux et d'incompréhensions politiques. C'est pourquoi il a proposé à la Nouvelle-Calédonie lors de l'actualisation de la loi organique en 2013 d'être associée aux négociations engagées par l'État intervenant dans les domaines de compétence du territoire. Pour la conférence de Rio+20 par exemple, la Nouvelle-Calédonie avait été partie prenante de la délégation française car cela

28. Au paragraphe 3.2.1., les relations internationales et régionales sont classées dans la catégorie des compétences partagées.

29. À ce titre, la Nouvelle-Calédonie a pu ouvrir et mener des négociations avec la CPS afin de redéfinir les conditions de l'établissement et les privilèges et immunités de celle-ci sur son territoire.

30. Il y a par exemple un agent en charge pour la province Sud.

31. Convention relative à l'accueil de délégués pour la Nouvelle-Calédonie au sein du réseau diplomatique de l'État dans le Pacifique, 26 janvier 2012. 
participait de la recherche d'une plus grande intégration régionale du territoire mais il n'y avait pas de base juridique pour justifier un tel choix. De même, il est apparu souhaitable de permettre au président du gouvernement ou à son représentant d'être associé et de participer aux travaux des organisations internationales auprès desquelles la France est membre mais pas ses territoires, lorsque les discussions portent sur des sujets relevant de leurs compétences. Pour les organisations régionales de pêche cela pourrait s'exprimer en confiant, dans certains cas, la représentation de la France à la NouvelleCalédonie, or aucune disposition juridique ne le permet explicitement.

Les quelques distorsions juridiques entre les compétences dévolues à la Nouvelle-Calédonie et la Polynésie française, le degré différent de bureaucratisation des administrations en charge des relations extérieures, les appétences diverses des responsables politiques et des intérêts parfois divergents doivent conduire l'État à s'interroger sur la nécessité de rechercher des places parfaitement identiques pour la Nouvelle-Calédonie et la Polynésie française dans toutes les organisations régionales voire sur la nécessité d'esquisser des politiques sous-régionales plus marquées dans le Pacifique Sud. Ce dimorphisme diplomatique Mélanésie-Polynésie, tout comme celui qu'impose les approches trans-Pacifique et intra-Pacifique, incite à décliner plus précisément les politiques sous-régionales françaises. Mais cette élasticité est susceptible de porter une moindre attention au monde micronésien auquel l'un des alliés majeurs, les États-Unis, est particulièrement attaché. Reste donc à savoir si une présence renforcée dans les organisations mini-multilatérales nées d'identités éclatées se traduira pour la France par une hiérarchisation plus affirmée dans ses approches des organisations sous-régionales, afin de tenir compte de leurs capacités d'action et de rayonnement propres qu'elles offriront à chacun de ses territoires océaniens.

\section{Le difficile accès à un espace économique océanien intégré}

Si l'État soutient très officiellement les demandes d'attribution d'un statut de plein droit pour la Nouvelle-Calédonie et la Polynésie française au sein des organisations politiques régionales comme le FIP et le FDIP, et finance pour partie les participations aux mécanismes mini-multilatéralistes - comme pour le Groupe des dirigeants polynésiens -, à cette construction étato-centrée de l'architecture régionale s'ajoute, de plus en plus, une mobilisation des acteurs de la société civile notamment économiques. La France se montre soucieuse de voir inscrits pleinement ses territoires dans les organisations sous-régionales socioprofessionnelles (par exemple, agriculture, pêche, tourisme...). Cependant, cet objectif ne peut être atteint que par la volonté des collectivités territoriales car nombre des organisations transnationales visées agissent dans des domaines de compétence déjà octroyés par l'État, or l'accession de la NouvelleCalédonie et/ou de la Polynésie française à des organisations économiques du Pacifique océanien n'est pas sans soulever de complexes enjeux notamment européens.

Les organisations les plus politiques ne sont pas sans promouvoir la coopération et l'intégration régionales en matière économique. L'article 2 de l'accord établissant le FIP (2005) et l'accord de libre-échange signé le 29 juillet 2004 entre les États membres du GFLM dont l'exécution constitue l'un des buts constitutifs de l'organisation mélanésienne le rappellent. Si l'adhésion de la Nouvelle-Calédonie et/ou la Polynésie aux organisations régionales d'intégration économique n'est en rien exclue, elle n'en demeure pas moins devoir être envisagée dans des conditions juridiquement contraintes, notamment au regard des engagements internationaux de la République. Les autorités territoriales doivent respecter les engagements en vigueur, pris au titre des territoires dans le cadre du GATT et de l'accord général sur le commerce des services (GATS). Cela impose de respecter les règles posées par le traité de la communauté européenne, telles que mises en œuvre par l'accord d'association des Ртом à l'Europe. Enfin, et surtout, cela nécessite une concertation avec les autorités communautaires pour s'assurer que la participation des territoires aux organisations régionales n'est pas susceptible de porter atteinte aux politiques développées dans le cadre de l'Union européenne. Autrement dit, toute initiative pouvant être prise par les autorités locales en application des compétences qui leur sont désormais dévolues dans les matières commerciale et douanière et visant par exemple à la libération des échanges de produits doit s'inscrire dans le contexte des règles posées par la décision d'association. Il s'agit de ne pas contrevenir à l'économie du système d'échange préférentiel mis en place par la Communauté au profit des РтOM et de ne pas établir de mécanismes discriminatoires entre les États de l'Union en matières commerciale et douanière. Dans la mesure où, en vertu de l'article 133 du traité sur le fonctionnement de l'Union européenne, la Communauté dispose d'une compétence exclusive en matière commerciale commune, la France est tenue d'envisager toute négociation d'adhésion aux organisations régionales à vocation économique et commerciale, à l'initiative des présidents des gouvernements des territoires, en concertation étroite avec les autorités de l'Union européenne. Une situation qui impose à l'État de conduire sa politique "Pacifique» en adéquation avec trois 
évolutions majeures de la politique européenne, l'avenir de l'accord de Cotonou, qui régit à l'heure actuelle les relations entre l'Union européenne et le groupe des États d'Afrique, des Caraïbes et du Pacifique (ACP) qui arrivera à échéance à la fin de l'année 2020, plus de synergies entre les politiques conduites avec les Etats du Pacifique du groupe ACP et les quatre PTOM du Pacifique et une attention aux impacts économiques sur les États et territoires océaniens des accords de libreéchange établis ou en cours de discussion avec les partenaires asiatiques, australien et néo-zélandais. Une raison supplémentaire pour intégrer institutionnellement l'Union européenne aux organisations intra-Pacifique.

\section{La fin des relations strictement étato-centrées et un plus grand contrôle démocratique}

Les critiques émises à l'encontre de la plupart des organisations régionales du Pacifique océanien portent aujourd'hui sur leur caractère strictement étato-centré. C'est pourquoi depuis quelques années, les ONG y réclament leur place et créent leurs propres plateformes d'échanges (par exemple l'association des femmes francophones océaniennes). Des législateurs, à l'instar du Néo-Zélandais Mike Moore (Moore, 1982) réclament eux l'instauration d'un Parlement du Pacifique à l'image du Parlement européen ou du Conseil nordique. Il s'agit de redonner $\mathrm{du}$ sens politique aux organisations régionales, une plus grande légitimité démocratique et d'associer les représentants des citoyens aux décisions visant à ordonnancer le Pacifique océanien. Mettre sur pied un dispositif de cette nature serait une gageüre en terme logistique puisqu'il s'agit de donner corps à un espace législatif s'étendant sur $10000 \mathrm{~km}$ d'Est en Ouest et 5000 du Nord au Sud. En attendant, les forums inter-parlementaires qui émergent depuis le début des années 90, rencontrent pour la Nouvelle-Calédonie, la Polynésie française, Wallis-et-Futuna l'écueil de la plupart des institutions intra-Pacifique puisqu' ils les excluent institutionnellement. L'espace géographique des échanges inter-parlementaires ne couvre soit qu'une partie du Pacifique - comme pour l'AsiaPacific Parliamentary Forum ${ }^{32}$ ou la Conférence de l'assemblée parlementaire du Commonwealth - soit la plupart des territoires à l'exclusion de la Françocéanie (par exemples l'Assemblée parlementaire conjointe ACP-UE, l'Association des bibliothécaires parlementaires, APLAP, le Forum parlementaire du Pacifique ou encore le Partenariat parlementaire du Pacifique). Cette situation donne le sentiment que les stratégies d'intégration régionale se confondent avec le périmètre des membres de droit du FIP et ne prennent pas l'espace Pacifique comme un tout. Une ambiguïté quel'on trouve dansles documents censés penser les processus d'intégration euxmêmes, Pacific Plan (2005, révisé en 2012) et Framework for Regionalism (2014), déclinant des priorités très générales et ne s'incarnant pas dans de véritables stratégies d'intégration au travers de politiques publiques coordonnées et adossées à des priorités pleinement partagées par les États et territoires océaniens. Cette absence de synergies entre les acteurs incitent les ONG, les chercheurs et les experts à demander plus de place dans les institutions régionales notamment celles ayant pour mandat de répondre aux principaux défis des politiques de développement. Les défis environnementaux étant les plus préoccupants à court, moyen et long terme, il n'est pas surprenant de voir s'esquisser pour y répondre une concurrence entre les opérateurs - CPS / PROE - et de nouvelles plateformes tribunitiennes associatives - Oceania 21 par exemple.

L'exigence grandissante de démocratisation des institutions régionales (Dearden, $2007: 10$ 12) est aussi une critique de leur peu d'efficacité intégratrice (Grynber, 2006). Dans ce contexte, le Pacifique insulaire se caractérise dorénavant par une volonté de s'arrimer aux puissances émergentes, par le resserrement des matrices sous-régionales y compris dans le champ économique (PICTA Trade in Goods Agreement, MSG Trade Agreement, Micronesian Trade Committee Memorandum of Understanding, Economic Partnership Agreement), l'émergence de forums mixtes associant les autorités gouvernementales, coutumières et des citoyens et la volonté de s'appuyer sur les sciences de la terre pour assurer un développement durable aux territoires. Dès lors, la nécessité de diplomaties scientifiques et la prise en compte des acteurs diversifiés des sociétés civiles sont le prochain enjeu pour tous les États souhaitant développer leur influence dans le Pacifique océanien. La modularité des diplomaties va donc s'accroître encore. La stratégie française sera donc axée sur la recherche d'une plus grande efficacité diplomatique de l'État en Asie-Pacifique, tout en étant la conséquence d'une atomisation de l'espace institutionnel régional, l'expression d'une influence grandissante des communautés d'outremer et de certains de leurs leaders sur la politique étrangère de l'hexagone. L'État fait le pari que sa politique "Pacifique" est la somme de ses stratégies sous-régionales, de celles de ses territoires et de sa politique asiatique mais également de ses acteurs infra-gouvernementaux, qu'ils soient académiques, associatifs, entrepreneuriaux ou encore sportifs. 


\section{BIBLIOGRAPHIE}

Aldrich Robert, 1990. The French Presence in the South Pacific (1842 - 1940), London, Macmillan.

-, 1993. France and the South Pacific since 1940, Honolulu, University of Hawaii Press.

Berg Eugène, 2013. France, l'horizon Pacifique, Géoéconomie 64, pp. 209-220.

Chen-YI Lin, 2010. The Security Implications of a Cross-Strait Competition in the South Pacific rom a Taiwanese Perspective, in Brady Anne-Maris, Looking North, Looking South 26, World Scientific Publishing, pp. 113-133.

Chesneaux Jean et Nic Maclellan, 1992. La France dans le Pacifique, De Bougainville à Mururuoa, Paris, La Découverte.

Chien-Peng Chung, 2010. China's Multilateral Co-Operation in Asia and the Pacific, London, Routledge.

Communiqué Conjoint entre la France et le Japon, 7 juin 2013, Un "partenariat d'exception " pour promouvoir la sécurité, la croissance, l'innovation et la culture (http://www.fr.embjapan.go.jp/actualite/pdf/Communique_ conjoint_France_Japon.pdf).

Cordonnier Isabelle, 1995. La France dans le Pacifique Sud. Approche géostratégique, Paris, Publisud.

Crocombe Ron, 1995. The Pacific Islands and the USA, Suva, University of the South Pacific.

Dearden Stephen, 2007. The European Union's Pacific Strategy and the New Framework for Pacific Regionalism, Manchester Metropolitan University (http://www.academia. edu/8611903/The_European_Unions_Pacific_Strategy_and_the_New_Framework_for_ Pacific_Regionalism)

Dobell Graeme, 2013 (18/09/). France in the South Pacific: Ambiguity and Amibition, ASPI Blog (http://aspistrategist.org.au/ france-in-the-pacific-ambiguity-and-ambition/).

Fisher Denise, 2013. France in the South Pacific: Power and Politics, Canberra, Australian National University.

Germain Philippe, 13 avril 2015. Déclaration de politique générale prononcée devant le congrès, au nom du $14^{\mathrm{e}}$ gouvernement de la Nouvelle-Calédonie (http://www.gouv.ncl portal/pls/portal/docs/1/29596253.PDF).

Godement François, 2014. France's "Pivot" to Asia, ECFr.

Grynberg Roman, 2006. Towards a New Pacific Regionalism, Asian Development Bank -
Commonwealth Secretariat (http://www.adb. $\mathrm{org} / \mathrm{sites} / \mathrm{default} /$ files/publication/28797/ pacific-regionalism-vol2.pdf).

Guilbert François, juin 2015. Le monde à la (re)découverte de l'Océanie, une "Pacificité " aux quatre vents, Lettre du réseau Asie.

Henningham Stephen, 1992. France and the South Pacific: A Contemporary History, Sydney, Allen \& Unwin.

HERMES, 2013. Hermès 65 : Le monde Pacifique dans la mondialisation, Paris, CNRs Éditions.

Hollande François, 19 novembre 2014. Discours devant la Communauté du Pacifique (http://www.elysee.fr/declarations/article/discours-devant-la-communaute-du-pacifique/).

Joint Statement by Indonesia and the Members of Melanesian Spearhead Group, 2014 (16/01). http://www.kemlu.go.id/ Documents/MSG\%202014/JOINT\%20 STATEMENT\%20INDONESIA\%20-\%20 MSG.pdf.

Larcher Serge (éd.), 2013. La France dans le Pacifique. Quelle vision pour le XXI siècle, Paris, Délégation sénatoriale à l'Outre-Mer.

LeChervy Christian, 2015a (sous presse). La France, une politique extérieure en phase avec une "Pacificité " polymorphe, Revue juridique politique et économique de Nouvelle-Calédonie 25, pp. 49-59.

—, 2015b (sous presse). Quels regards porter sur la politique chinoise dans le Pacifique-océanien?, Revue de Défense nationale 781, pp.71-78.

Methven Philip, 1986. The French Approach to Regional Security in the South Pacific, Canberra, Australian National University.

Ministère de la Défense, 2013. La France et la sécurité en Asie-Pacifique.

Mohamed-Gaillard Sarah, 2010. L'archipel de la puissance ?, La politique de la France dans le Pacifique Sud de 1946 à 1998, Bruxelles, PIE Peter Lang.

Moore Mike, 1982. A Pacific Parliament. A Pacific Idea: An Economic and Political Community of the South Pacific, Suva, University of the South Pacific.

Mrgudovic Nathalie, 2008. La France dans le Pacifique Sud 1966-2006, enjeux et mutations, thèse, Université Bordeaux IV.

Polling Gregory, 2015 (May). The Lessons in Fiji's Campaign to Change the PIF, Pacific Partners Outlook V/4, pp.1-3 (http://csis.org/ files/publication/150507_PacificPartners_ Vol_5_Issue_4.pdf). 
Annexe 1. - Schématisation des relations internationales du forum des îles du Pacifique (@ C. Lechervy)

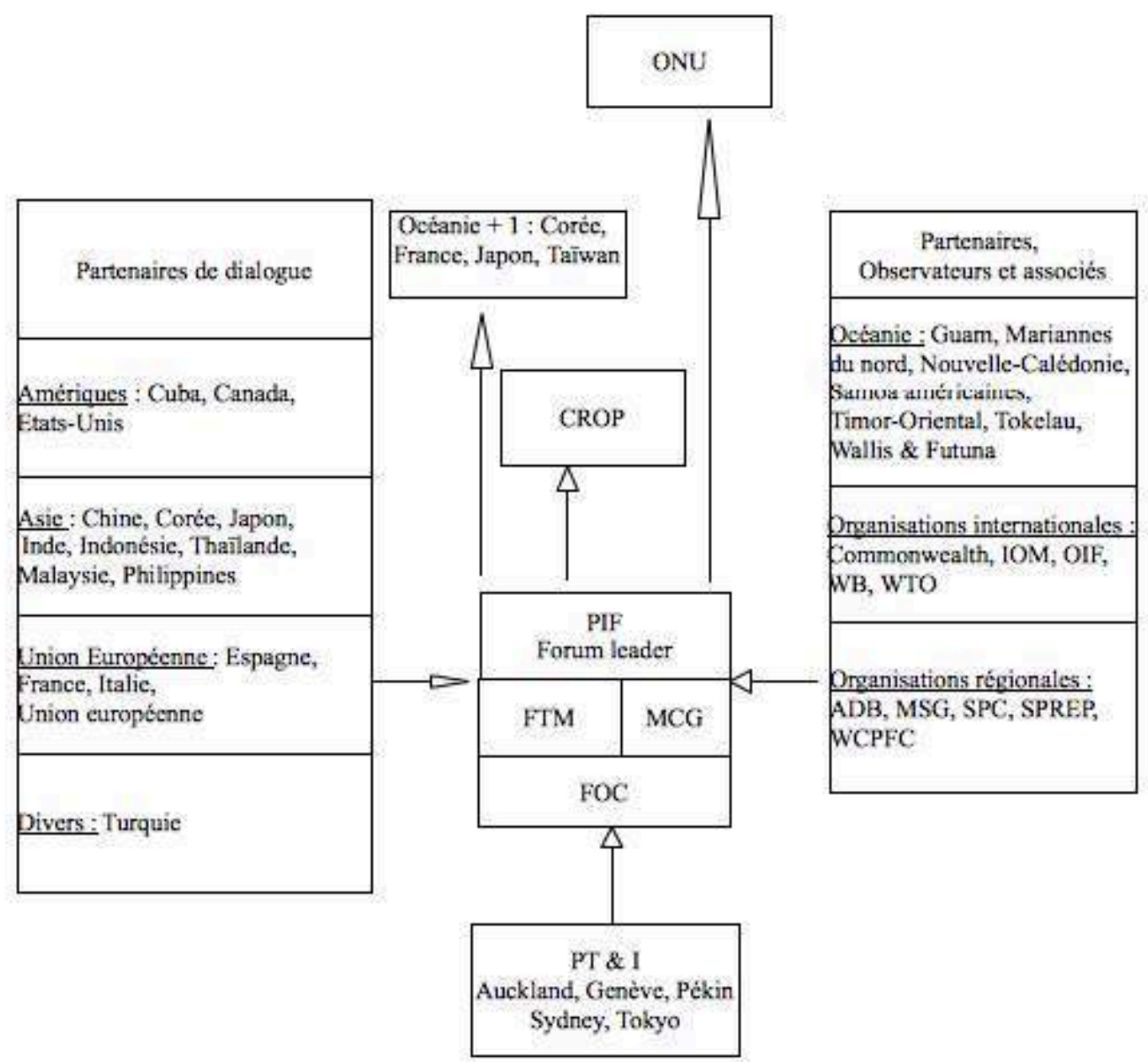




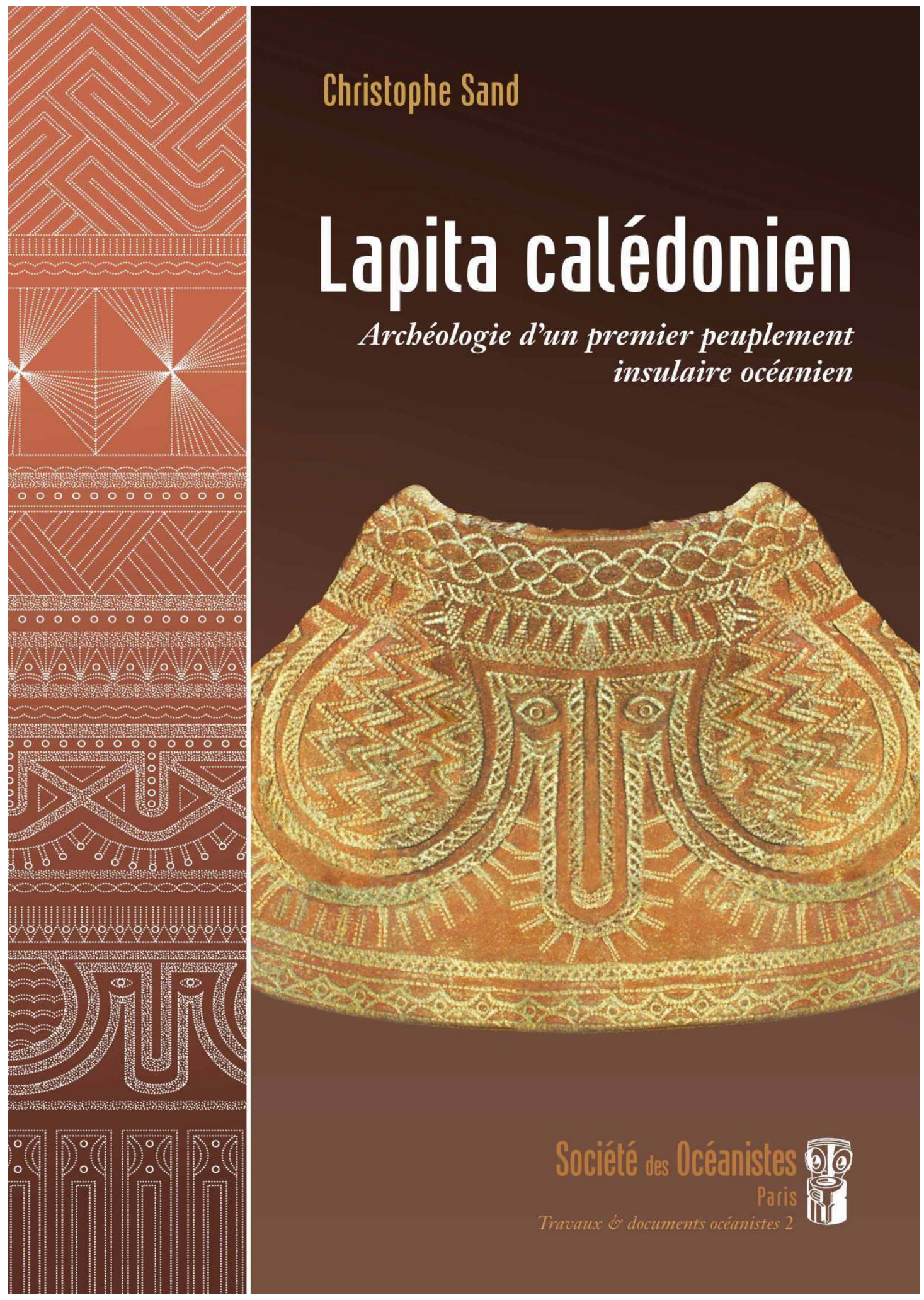

2010, 288 p., bibliographie, index, nombreuses illustrations couleur - $38 €$. En vente sur http://oceanistes.org/oceanie/spip.php?rubrique29 avec paiement en ligne et en version électronique sur http://books.openedition.org/sdo/1128-29,99€. 